\title{
Occurrence and Distribution of Some Organic Contaminants in Sediments from Olt River, Romania $^{+}$
}

\author{
Corina Teodora Ciucure, Elisabeta-Irina Geană *, Diana Ionela Popescu, Alin Chitu, \\ Andreea-Maria Rădulescu and Roxana Elena Ionete
}

National Research and Development Institute for Cryogenics and Isotopic Technologies-ICSI Rm. Valcea, Uzinei Street No. 4, Rm., 240050 Valcea, Romania; corina.ciucure@icsi.ro (C.T.C.);

diana.popescu@icsi.ro (D.I.P.); alin.chitu@icsi.ro (A.C.); andreea.iordache@icsi.ro (A.-M.R.);

roxana.ionete@icsi.ro (R.E.I.)

* Correspondence: irina.geana@icsi.ro

+ Presented at the 16th International Symposium "Priorities of Chemistry for a Sustainable Development" PRIOCHEM, Bucharest, Romania, 28-30 October 2020.

Published: 10 November 2020

Keywords: environmental contamination; organic pollutants; surface sediments

Organic contaminants (OCs) accumulate in the environment as a result of anthropogenic activities (industrial, agricultural, and household activities as well as auto traffic) and produce negative effects on the environment and humans. Due to their low solubility in water and their affinity for suspended particles, OCs tend to adhere to suspended particles and subsequently remain in sediments [1]. The purpose of this study was to analyze the distribution of the fifteen polycyclic aromatic hydrocarbons (PAHs), twelve polychlorinated biphenyls (PCBs), and nine organochlorine pesticides (OCPs) in surface sediments on the middle and lower course of the Olt river, Romania, and to determine their sources based on some diagnostic ratios combined with statistical approach.

Sediment samples were collected on the middle and lower basin of the Olt river, including the accumulation lakes and tributaries on its course (28 sampling sites), during three sampling campaigns, in 2019. Microwave extraction protocol was used in order to extract the analytes from the sediment samples, followed by quantitative determinations of PAHs by HPLC-FLD and PCBs and OCPs by GC-ECD. Some PAHs and OCPs diagnostic ratios were used to suggest the possible contamination sources. A principal component analysis (PCA) was used in order to distinguish the possible sources of specific contamination and to highlight the locations with considerable ecological risk (Figure 1).

Although OCs levels in sediment samples are moderate, some sampling sites show exceeded values for $\Sigma 10 \mathrm{PAH}$ and $\Sigma \mathrm{PCB}$, indicating high contamination. Most of the sites were characterized by Phe/An values $<10$ and Flt/Py values $<1$, which correspond to strong pyrolytic input, while some sites show Phe/An values $>10$ and Flt/Py values $>1$, indicating a petrogenic contamination.

This study provided the first detailed analysis of the distribution of some OCs in surface sediments collected on the middle and lower course of the river Olt, Romania. A high spatial variability was observed for the studied OCs, with the highest concentrations of PAHs and PCBs near industrial areas and urban agglomerations, while OCPs were identified mainly in rural areas. 

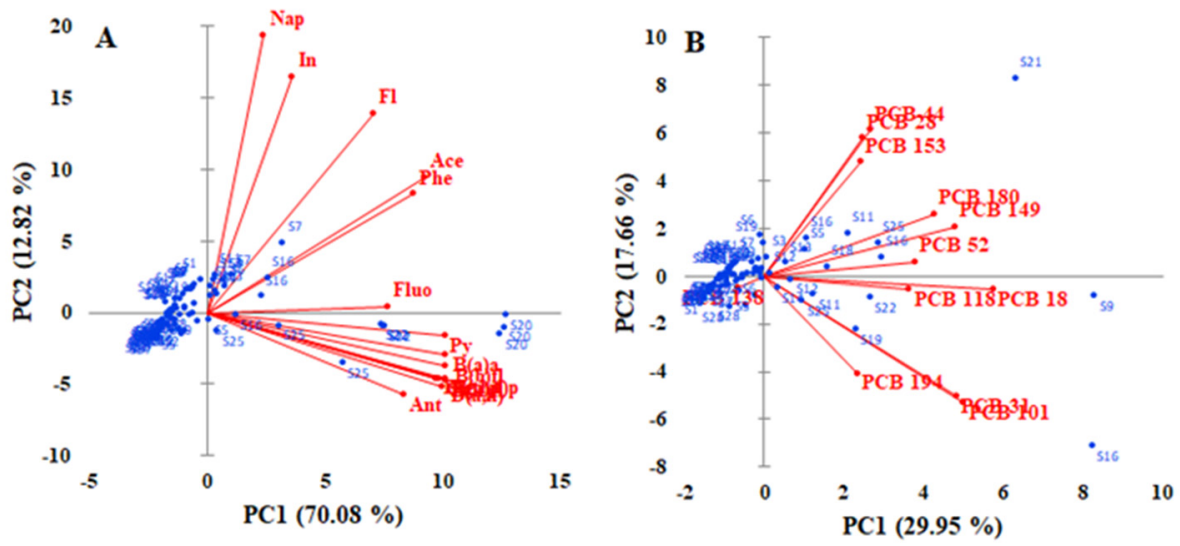

Figure 1. Principal component analysis (PCA) analysis for concentrations of: polycyclic aromatic hydrocarbons (PAHs) (A) and of polychlorinated biphenyls (PCBs) (B) in surface sediments of the Olt river.

Acknowledgments: Romanian Ministry of Research and Innovation, grant number PN 19110303 "Advanced techniques for identifying sources of contamination and biochemical reactions in aquatic ecosystems".

\section{References}

1. Mitra, S.; Corsolini, S.; Pozo, K.; Audy, O.; Sarkar, S.; Biswas, J.K. Characterization, source identification and risk associated with polyaromatic and chlorinated organic contaminants (PAHs, PCBs, PCBzs and OCPs) in the surface sediments of Hooghly estuary, India. Chemosphere 2019, 221, 154-165.

Publisher's Note: MDPI stays neutral with regard to jurisdictional claims in published maps and institutional affiliations.

(C) 2020 by the authors. Licensee MDPI, Basel, Switzerland. This article is an open access article distributed under the terms and conditions of the Creative Commons Attribution (CC BY) license (http://creativecommons.org/licenses/by/4.0/). 